\title{
Shear stresses in rectangular panels of ship structures in the calculations according to Reissner theory
}

\author{
Mikhail Sukhoterin ${ }^{1, *}$, Sergey Baryshnikov ${ }^{1}$, Elena Rasputina $^{1}$ and Natalia Pizhurina ${ }^{1}$ \\ ${ }^{1}$ Admiral Makarov State University of Maritime and Inland Shipping, 5/7 Dvinskaya street, 198035, \\ St. Petersburg, Russia
}

\begin{abstract}
This study calculates and analyzes torsion moments of a rectangular panel with clamped edges as an element of ship structures under the action of uniform pressure with allowance for transverse shear deformation and examines the contribution of the corresponding shear stresses to the general stress state. In order to solve this problem, the method of infinite superposition of corrective functions of bending and stresses is applied. It involves an iterative process of mutually correcting the discrepancies from the said functions while meeting all boundary conditions. A particular solution for the bending function in the form of a quadratic polynomial is chosen as the initial approximation. It is established that torsion moment series diverge at the corner points of the plate going into infinity, which yields infinite values for the shear stresses at these points as well. Results of torsion moment calculation for square plates with different width ratios are provided. A 3D distribution diagram of moments is obtained. The computational experiment confirms the correctness of theoretical conclusions about infinite torsion moments at the corner points of the plate. Comparison with bending moments shows that torsion moments cannot be ignored during the assessment of the stress-strain state. The behavior of torsion moments near the corner points is qualitatively different from the simplified Kirchhoff theory, where they turn into zero.
\end{abstract}

\section{Introduction}

Modern ship structures widely use metallic and non-metallic materials with high interlaminar shear strength. Panels (plates) that serve as the basic elements of plating, deck sheathing, bulkheads, etc. are often made of such materials. This requires using more accurate methods of analysis during calculation that would reduce the weight of the structure without compromising its strength.

The classical (simplified) theory of thin plates based on the hypotheses of Kirchhoff and Love yields a significant error near the edges of the plate, in areas of steep changes in boundary conditions and points of application of concentrated forces, and during the calculation of medium thick plates.

\footnotetext{
* Corresponding author: mv@sukhoterin.com
} 
The linear plate-bending theory, which qualitatively specifies the classical theory, was first introduced by Reissner [1], who obtained new differential equations of plate bending and the corresponding boundary conditions with allowance for the effects of the transverse shear. The system of two differential equations consists of a fourth-order equation with respect to the bending function and an additional second-order equation with respect to the stress function. This makes it possible to meet three boundary conditions (instead of two in the classical theory) on each edge of the plate.

The above theory and the similar shear theories that followed are called Reissner Mindlin [2] - Timoshenko [3] theories due to their similarity. For example, the difference between the Reissner and Mindlin theories virtually comes down to transverse shear ratio values, which are not that different.

Also worth mentioning are the variants of the shear theory from recent works [4-12].

There are few known results on the problem of bending of a rectangular panel with clamped edges using shear theories due to the complexity of the corresponding boundary problem. Known works [12-19] mainly contain numerical results for bending or, less commonly, calculate bending moments and shear forces at specific points. However, these works lack calculations of torsion moments, which can significantly affect shear stresses as elements of the general stress state.

It should be noted that during the calculation of plates according to the simplified Kirchhoff theory researchers rarely calculate torsion moments because they consider the contribution of the corresponding shear stresses to the general stress state to be insignificant. However, there may occur a redistribution of shear stresses at the corner points of the contour, which is ignored by the Kirchhoff theory. This can change the qualitative picture of the stress state near these points.

The main aim of this study is to obtain reliable numerical results on the torsion moments of a rectangular panel with clamped edges with allowance for transverse shear deformation according to the Reissner theory and to assess the impact of the corresponding shear stresses on the general stress state.

\section{Methods and Materials}

The system of differential equations in dimensionless form is written as follows [3].

$$
\begin{aligned}
& \nabla^{2} \nabla^{2} w(x, y)=-1, \\
& \psi(x, y)-\alpha \nabla^{2} \psi(x, y)=0 .
\end{aligned}
$$

where $\nabla^{2}$ is the Laplace bivariate operator; $w(x, y)=W /(q b 4 / D)$ is the relative bending of the points of the middle surface of the plate; $\mathrm{x}=\mathrm{X} / \mathrm{b}, \mathrm{y}=\mathrm{Y} / \mathrm{b}$ are the relative coordinates; $\mathrm{W}$ is the absolute bending of the corresponding points; $q$ is the transverse distributed load; $b$ is the plate width; $\mathrm{D}=\mathrm{EH} 3 /[12(1-\mathrm{v} 2)]$ is the bending rigidity of the plate; $\mathrm{E}$ is the Young modulus; $\mathrm{H}$ is the plate thickness; $v$ is the Poisson ratio; $\psi(\mathrm{x}, \mathrm{y})=\Psi(\mathrm{X}, \mathrm{Y}) / \mathrm{qb} 2$ is the dimensionless stress function; $\Psi(\mathrm{X}, \mathrm{Y})$ is the stress function; $\alpha=\mathrm{h} 2 / 10$ is the shear ratio; $\mathrm{h}=$ $\mathrm{H} / \mathrm{b}$ is the relative plate thickness.

A plate with clamped edges often serves as the computational model for rectangular elements of ship structures. Boundary conditions consist in the absence of deflections and angles of clamped edges $x= \pm \gamma / 2, y= \pm 1 / 2$

$$
w=0, \quad \varphi_{x}=0, \quad \varphi_{y}=0 .
$$

where $\gamma=a / b$ is the aspect ratio of the plate; $\varphi_{x}, \varphi_{y}$ are the section angles $x=$ const, $y=$ const: 


$$
\begin{gathered}
\varphi_{x}=\frac{\partial}{\partial x}\left(w+\alpha_{1} \nabla^{2} w\right)-\alpha_{1} \frac{\partial \psi}{\partial y} ; \quad \varphi_{y}=\frac{\partial}{\partial y}\left(w+\alpha_{1} \nabla^{2} w\right)+\alpha_{1} \frac{\partial \psi}{\partial x} \\
\left(\alpha_{1}=\frac{2 \alpha}{1-v}\right) .
\end{gathered}
$$

According to [3], torsion moments are related to the bending and stress functions through the following formula:

$$
M_{x y}=(1-v) \frac{\partial^{2} w}{\partial x \partial y}+2 \alpha \frac{\partial^{2}}{\partial x \partial y} \nabla^{2} w-\alpha\left(\frac{\partial^{2} \psi}{\partial y^{2}}-\frac{\partial^{2} \psi}{\partial x^{2}}\right) .
$$

Here, the torsion moment is assigned to value $q b^{2}$.

It is necessary to find an analytical expression for torsion moments at each point in order to determine the corresponding shear stresses. According to (4), this involves solving the basic problem of finding unknown deflection and stress functions.

According to the Reissner shear model, stresses along the thickness of the plate are determined using the following formulas:

$$
\begin{gathered}
\sigma_{x}=\frac{12 M_{x} z}{h^{3}}, \quad \sigma_{y}=\frac{12 M_{y} z}{h^{3}}, \tau_{x y}=\frac{12 M_{x y} z}{h^{3}}, \\
\tau_{y z}=\frac{3 Q_{y}}{2 h}\left[1-\left(\frac{2 z}{h}\right)^{2}\right], \quad \tau_{z x}=\frac{3 Q_{x}}{2 h}\left[1-\left(\frac{2 z}{h}\right)^{2}\right],
\end{gathered}
$$

where $-h / 2 \leq z \leq h / 2$ is the applicate of a random point of the plate. Formulas (5) feature linear bending moment $M_{x}, M_{y}$, torsion moment $M_{x y}$, shear forces $Q_{x}, Q_{y}$. The maximum values of normal stresses $\sigma_{x}, \sigma_{y}$, and shear stresses $\tau_{x y}$ will be valid with $z=h / 2$

$$
\sigma_{x \max }=\frac{6 M_{x}}{h^{2}}, \quad \sigma_{y \max }=\frac{6 M_{y}}{h^{2}}, \tau_{x y \max }=\frac{6 M_{x y}}{h^{2}},
$$

while the maximum values of shear stresses $\tau_{y z}, \tau_{z x}$ will be valid with $z=0$ :

$$
\tau_{y z \max }=\frac{3 Q_{y}}{2 h}, \quad \tau_{z x \max }=\frac{3 Q_{x}}{2 h} .
$$

The problem of determining bending functions $w(x, y)$ and stress functions $\psi(x, y)$ are solved in works [20] using superposition of corrective functions. The required bending and stress functions are represented by the following expressions:

$$
\begin{gathered}
w(x, y)=w_{0}(x, y)+\sum_{n=1}^{\infty} w_{1 n}(x, y)+w_{2 n}(x, y), \\
\psi(x, y)=\sum_{n=1}^{\infty} \psi_{1 n}(x, y)+\psi_{2 n}(x, y),
\end{gathered}
$$

where

$$
w_{0}(x, y)=-\frac{1}{8}\left(x^{2}-\frac{\gamma^{2}}{4}\right)\left(y^{2}-\frac{1}{4}\right)
$$


is the initial deflection, while corrective functions are selected in the form of hyperbolictrigonometric series.

$$
\begin{gathered}
w_{1 n}(x, y)=\sum_{k=1,3, \ldots}^{\infty}(-1)^{k^{*}} \frac{A_{k n}}{\cosh \tilde{\lambda}_{k}}\left(x \sinh \lambda_{k} x-\frac{\gamma}{2} \tanh \tilde{\lambda}_{k} \cosh \lambda_{k} x\right) \cos \lambda_{k} y, \\
w_{2 n}(x, y)=\sum_{s=1,3, \ldots}^{\infty}(-1)^{s^{*}} \frac{B_{s n}}{\cosh \tilde{\mu}_{s}}\left(y \sinh \mu_{s} y-\frac{1}{2} \tanh \tilde{\mu}_{s} \cosh \mu_{s} y\right) \cos \mu_{s} x, \\
\psi_{1 n}(x, y)=\sum_{k=1,3, \ldots}^{\infty}(-1)^{k^{*}} C_{k n} \sinh \beta_{k} x \sin \lambda_{k} y, \\
\psi_{2 n}(x, y)=\sum_{s=1,3, \ldots}^{\infty}(-1)^{s^{*}} D_{s n} \sinh \xi_{s} y \sin \mu_{s} x .
\end{gathered}
$$

Here, $\mathrm{A}_{\mathrm{kn}}, B_{s n}, C_{k n}, D_{s n}$ are the undetermined ratios that are found in pairs when boundary conditions on parallel edges are met,

$$
\begin{gathered}
\lambda_{k}=k \pi, \quad \mu_{s}=\frac{s \pi}{\gamma}, \quad k^{*}=\frac{k+1}{2}, \quad s^{*}=\frac{s+1}{2} . \\
\tilde{\lambda}_{k}=\frac{\lambda_{k} \gamma}{2}, \quad \tilde{\mu}_{s}=\frac{\mu_{s}}{2}, \quad \beta_{k}=\sqrt{\lambda_{k}^{2}+\frac{1}{\alpha}}, \quad \xi_{s}=\sqrt{\mu_{s}^{2}+\frac{1}{\alpha}} .
\end{gathered}
$$

The essence of the method is that all boundary conditions of the problem are met as a result of an infinite iterative process of eliminating shear deformations (discrepancies) from initial function (8) and from each pair of corrective functions (9), (11) and (10, (12), which result in discrepancies on the other two edges as they eliminate discrepancies on two parallel edges through undetermined ratios.

Initial bending (8) is a particular solution of the first differential equation (1) that "automatically" ensures zero bending of clamped edges. The corrective bending functions are biharmonic, i.e. they satisfy the corresponding homogeneous equation for the first equation (1). They also ensure zero bending at the plate contour. The corrective stress function satisfy the second equation (1).

Work [20] calculates deflections, bending moments, and shear forces for different relative thicknesses of a square uniformly loaded plate and provides detailed tables. Torsion moments are not calculated as it is assumed that the corresponding shear stresses are minor compared with normal stresses. However, the subsequent analysis of the expression for torsion moments shows specific aspects of their behavior and effect on the stress state of the plate, particularly near the corner points. The numerical experiment confirms theoretical conclusions and provides a complete picture of torsion moment distribution in the plate. Below we provide the main results of the study.

By substituting the found bending functions (6) and (7) into formula (4) for torsion moments we obtain the final expression used in calculations:

$$
\begin{gathered}
M_{x y}=-\frac{1-v}{2} x y-\sum_{k=1,3, \ldots}^{\infty}(-1)^{k^{*}}\left\{\left(\left[(1-v)\left(1-\tilde{\lambda}_{k} \tanh \tilde{\lambda}_{k}\right)+4 \alpha \lambda_{k}^{2}\right] \sinh \lambda_{k} x+(1-v) \lambda_{k} x \cosh \lambda_{k} x\right.\right. \\
\left.\left.-2 \alpha \frac{\lambda_{k} \cosh \tilde{\lambda}_{k}}{\beta_{k} \cosh \tilde{\beta}_{k}}\left(\lambda_{k}^{2}+\beta_{k}^{2}\right) \sinh \beta_{k} x\right) \frac{\lambda_{k} A_{k \Sigma}}{\cosh \tilde{\lambda}_{k}}+2 \alpha \frac{\lambda_{k}^{2}+\beta_{k}^{2}}{\lambda_{k}^{2} \beta_{k} \cosh \tilde{\beta}_{k}} \sinh \beta_{k} x\right\} \sin \lambda_{k} y
\end{gathered}
$$




$$
\begin{gathered}
-\sum_{s=1,3, \ldots}^{\infty}(-1)^{s^{*}}\left\{\left(\left[(1-v)\left(1-\tilde{\mu}_{s} \tanh \tilde{\mu}_{s}\right)+4 \alpha \mu_{s}^{2}\right] \sinh \mu_{s} y+(1-v) \mu_{s} y \cosh \mu_{s} y\right.\right. \\
\left.\left.-2 \alpha \frac{\mu_{s} \cosh \tilde{\mu}_{s}}{\xi_{s} \cosh \tilde{\xi}_{s}}\left(\mu_{s}^{2}+\xi_{s}^{2}\right) \sinh \xi_{s} y\right) \frac{\mu_{s} B_{s \Sigma}}{\cosh \tilde{\mu}_{s}}+\frac{2 \alpha}{\gamma} \frac{\mu_{s}^{2}+\xi_{s}^{2}}{\mu_{s}^{2} \xi_{s} \cosh \tilde{\xi}_{s}} \sinh \xi_{s} y\right\} \sin \mu_{s} x(14)
\end{gathered}
$$

where $A_{k \Sigma}=A_{k 1}+A_{k 2}+\ldots+A_{k n} ; \ldots ; B_{s \Sigma}=B_{s 1}+B_{s 2}+\ldots+B_{s n}$ are the cumulative ratios for all iterations.

It should be noted that ratios $C_{k n}, D_{s n}$, which are parts of stress functions, are expressed through "basic" ratios $A_{k n}, B_{s n}$ providing that boundary conditions are met.

The expression of torsion moments (14) is an odd function of both variables. Therefore, it is enough to obtain numerical results for the first quarter of the plate.

It is easy to see that in the center of the plate torsion moments turn into zero. Let us find their expression at the corner points, particularly at point $(0.5 \gamma, 0.5)$. After simplification, we get

$$
\begin{aligned}
\left.M_{x y \mid}\right|_{\substack{x=\gamma / 2 \\
y=1 / 2}}= & -\frac{1-v}{2} \frac{\gamma}{4}+\sum_{k=1,3, \ldots}^{\infty}\left\{\left(\left[(1-v)\left(1+\frac{\tilde{\lambda}_{k}}{\cosh \tilde{\lambda}_{k}}\right)+4 \alpha \lambda_{k}^{2}\right] \tanh \tilde{\lambda}_{k}\right.\right. \\
& \left.\left.-2 \alpha \frac{\lambda_{k}}{\beta_{k}}\left(\lambda_{k}^{2}+\beta_{k}^{2}\right) \tanh \tilde{\beta}_{k}\right) \lambda_{k} A_{k \Sigma}+2 \alpha \frac{\lambda_{k}^{2}+\beta_{k}^{2}}{\lambda_{k}^{2} \beta_{k}} \tanh \tilde{\beta}_{k}\right\} \\
& +\sum_{s=1,3, \ldots}^{\infty}\left\{\left(\left[(1-v)\left(1+\frac{\tilde{\mu}_{s}}{\cosh \tilde{\mu}_{s}}\right)+4 \alpha \mu_{s}^{2}\right] \tanh \tilde{\mu}_{s}\right.\right. \\
& \left.\left.-2 \alpha \frac{\mu_{s}}{\xi_{s}}\left(\mu_{s}^{2}+\xi_{s}^{2}\right) \tanh \tilde{\xi}_{s}\right) \mu_{s} B_{s \Sigma}+\frac{2 \alpha}{\gamma} \frac{\mu_{s}^{2}+\xi_{s}^{2}}{\mu_{s}^{2} \xi_{s}} \tanh \tilde{\xi}_{s}\right\}
\end{aligned}
$$

Using formulas (13) it is possible to find the limit of the following components of this expression:

$$
\begin{gathered}
\lim _{k \rightarrow \infty}\left[4 \alpha \lambda_{k}^{2} \tanh \tilde{\lambda}_{k}-2 \alpha \frac{\lambda_{k}}{\beta_{k}}\left(\lambda_{k}^{2}+\beta_{k}^{2}\right) \tanh \tilde{\beta}_{k}\right] \\
=\lim _{k \rightarrow \infty}\left[4 \alpha \lambda_{k}^{2} \tanh \tilde{\lambda}_{k}\left(1-\frac{\lambda_{k}}{\beta_{k}} \frac{\tanh \tilde{\beta}_{k}}{\tanh \tilde{\lambda}_{k}}\right)-2 \frac{\lambda_{k}}{\beta_{k}} \tanh \tilde{\beta}_{k}\right]=\frac{2}{1-v}-2=\frac{2 v}{1-v} .
\end{gathered}
$$

Ratios $A_{k}$ and $B_{s}$ have estimates obtained in [20]: $A_{k n}=O\left(1 / k^{2}\right), B_{s n}=O\left(1 / s^{2}\right)$ for any iteration. Then the general first-order term in (15) has $k$-index exponent $O(1 / k)$. Similarly, the second-order term in (15) has exponent $O(1 / s)$.

This means that series in (15) diverge at the corner point and yield infinite torsion moments and, consequently, shear stresses. 


\section{Results}

Numerical results for torsion moments are obtained for a rectangular plate $(\gamma=1)$ with fully clamped edges (CCCC plate according to the international nomenclature) under the effect of uniform pressure according to the refined Reissner theory. Calculations are made using a computer program compiled in the Maple system. The Poission number is taken equal to 0.3. The number of terms held in series (14) is taken equal to 299, the number of iterations is 10 . After the 10th iteration, discrepancies in the fulfillment of boundary conditions, which are printed after each iteration, are practically zero. The relative thickness is taken equal to $h=$ $0.01,0.05,0.1,0.2$, and 0.3 . Tables $1-5$ show torsion moment values at different points of the plate for the given relative thicknesses. Computational points are condensed near the contour in order to examine the effect of the plate edge. Fig. 1-5 show the corresponding torsion moment diagrams.

Table 1. Torsion moment values in relation to value $-q b^{2} \times 10^{-6}$ for a square Reissner plate with relative thickness $h=0.01$ (CCCC plate, $q=$ const).

\begin{tabular}{|c|c|c|c|c|c|c|c|c|c|c|}
\hline \multirow{2}{*}{$\boldsymbol{y}$} & \multicolumn{10}{|c|}{$\boldsymbol{x}$} \\
\cline { 2 - 12 } & 0 & 0.1 & 0.2 & 0.3 & 0.4 & 0.42 & 0.44 & 0.46 & 0.48 & 0.5 \\
\hline 0 & 0 & 0 & 0 & 0 & 0 & 0 & 0 & 0 & 0 & 0 \\
\hline 0.1 & 0 & 1721 & 3109 & 3730 & 2965 & 2576 & 2091 & 1504 & 809 & 195 \\
\hline 0.2 & 0 & 3109 & 5652 & 6860 & 5565 & 4862 & 3972 & 2877 & 1560 & 380 \\
\hline 0.3 & 0 & 3730 & 6860 & 8483 & 7095 & 6255 & 5166 & 3790 & 2085 & 516 \\
\hline 0.4 & 0 & 2965 & 5565 & 7095 & 6109 & 5417 & 4508 & 3346 & 1877 & 478 \\
\hline 0.42 & 0 & 2576 & 4862 & 6255 & 5417 & 4797 & 3980 & 2943 & 1650 & 420 \\
\hline 0.44 & 0 & 2091 & 3972 & 5166 & 4508 & 3980 & 3280 & 2400 & 1323 & 335 \\
\hline 0.46 & 0 & 1504 & 2877 & 3790 & 3346 & 2943 & 2400 & 1710 & 900 & 214 \\
\hline 0.48 & 0 & 809 & 1560 & 2085 & 1877 & 1650 & 1323 & 900 & 412 & 65 \\
\hline 0.5 & 0 & 195 & 380 & 516 & 478 & 420 & 335 & 214 & 65 & -21 \\
\hline
\end{tabular}

Table 2. Torsion moment values in relation to value $-q b^{2} \times 10^{-6}$ for a square Reissner plate with relative thickness $h=0.05$ (CCCC plate, $q=$ const).

\begin{tabular}{|c|c|c|c|c|c|c|c|c|c|c|}
\hline \multirow{2}{*}{$\boldsymbol{y}$} & \multicolumn{10}{|c|}{$\boldsymbol{x}$} \\
\cline { 2 - 11 } & 0 & 0.1 & 0.2 & 0.3 & 0.4 & 0.42 & 0.44 & 0.46 & 0.48 & 0.5 \\
\hline 0 & 0 & 0 & 0 & 0 & 0 & 0 & 0 & 0 & 0 & 0 \\
\hline 0.1 & 0 & 1684 & 3042 & 3652 & 2911 & 2535 & 2076 & 1555 & 1063 & 962 \\
\hline 0.2 & 0 & 3042 & 5524 & 6699 & 5439 & 4762 & 3922 & 2958 & 2043 & 1850 \\
\hline 0.3 & 0 & 3652 & 6700 & 8257 & 6883 & 6073 & 5048 & 3848 & 2689 & 2452 \\
\hline 0.4 & 0 & 2911 & 5440 & 6883 & 5885 & 5218 & 4364 & 3349 & 2359 & 2158 \\
\hline 0.42 & 0 & 2535 & 4762 & 6073 & 5218 & 4625 & 3861 & 2957 & 2075 & 1895 \\
\hline 0.44 & 0 & 2076 & 3922 & 5049 & 4364 & 3862 & 3213 & 2445 & 1701 & 1546 \\
\hline 0.46 & 0 & 1555 & 2958 & 3848 & 3350 & 2957 & 2445 & 1836 & 1248 & 1125 \\
\hline 0.48 & 0 & 1063 & 2043 & 2689 & 2359 & 2076 & 1701 & 1248 & 785 & 668 \\
\hline 0.5 & 0 & 962 & 1851 & 2452 & 2159 & 1895 & 1547 & 1125 & 668 & -111 \\
\hline
\end{tabular}


Table 3. Torsion moment values in relation to value $-q b^{2} \times 10^{-6}$ for a square Reissner plate with relative thickness $h=0.1$ (CCCC plate, $q=$ const).

\begin{tabular}{|c|c|c|c|c|c|c|c|c|c|c|}
\hline \multirow{2}{*}{$\boldsymbol{y}$} & \multicolumn{10}{|c|}{$\boldsymbol{x}$} \\
\cline { 2 - 11 } & 0 & 0.1 & 0.2 & 0.3 & 0.4 & 0.42 & 0.44 & 0.46 & 0.48 & 0.5 \\
\hline 0 & 0 & 0 & 0 & 0 & 0 & 0 & 0 & 0 & 0 & 0 \\
\hline 0.1 & 0 & 1584 & 2859 & 3430 & 2799 & 2507 & 2185 & 1880 & 1685 & 1782 \\
\hline 0.2 & 0 & 2859 & 5182 & 6270 & 5186 & 4661 & 4078 & 3521 & 3163 & 3348 \\
\hline 0.3 & 0 & 3430 & 6270 & 7689 & 6490 & 5862 & 5155 & 4470 & 4026 & 4268 \\
\hline 0.4 & 0 & 2799 & 5186 & 6490 & 5591 & 5063 & 4458 & 3866 & 3483 & 3704 \\
\hline 0.42 & 0 & 2507 & 4661 & 5862 & 5063 & 4578 & 4018 & 3470 & 3118 & 3324 \\
\hline 0.44 & 0 & 2185 & 4078 & 5155 & 4458 & 4018 & 3505 & 2988 & 2656 & 2846 \\
\hline 0.46 & 0 & 1880 & 3521 & 4470 & 3866 & 3470 & 2988 & 2476 & 2110 & 2268 \\
\hline 0.48 & 0 & 1685 & 3163 & 4026 & 3483 & 3118 & 2656 & 2110 & 1562 & 1563 \\
\hline 0.5 & 0 & 1782 & 3348 & 4268 & 3704 & 3324 & 2846 & 2268 & 1563 & -712 \\
\hline
\end{tabular}

Table 4. Torsion moment values in relation to value $-q b^{2} \times 10^{-6}$ for a square Reissner plate with relative thickness $h=0.2$ (CCCC plate, $q=$ const $)$.

\begin{tabular}{|c|c|c|c|c|c|c|c|c|c|c|}
\hline \multirow{2}{*}{$\boldsymbol{y}$} & \multicolumn{10}{|c|}{$\boldsymbol{x}$} \\
\cline { 2 - 12 } & 0 & 0.1 & 0.2 & 0.3 & 0.4 & 0.42 & 0.44 & 0.46 & 0.48 & 0.5 \\
\hline 0 & 0 & 0 & 0 & 0 & 0 & 0 & 0 & 0 & 0 & 0 \\
\hline 0.1 & 0 & 1331 & 2400 & 2917 & 2709 & 2612 & 2528 & 2483 & 2517 & 2687 \\
\hline 0.2 & 0 & 2400 & 4344 & 5313 & 4967 & 4796 & 4644 & 4564 & 4628 & 4945 \\
\hline 0.3 & 0 & 2917 & 5313 & 6558 & 6177 & 5970 & 5788 & 5694 & 5784 & 6196 \\
\hline 0.4 & 0 & 2709 & 4967 & 6177 & 5704 & 5448 & 5217 & 5093 & 5186 & 5630 \\
\hline 0.42 & 0 & 2612 & 4796 & 5970 & 5448 & 5155 & 4873 & 4696 & 4758 & 5204 \\
\hline 0.44 & 0 & 2528 & 4644 & 5788 & 5217 & 4873 & 4507 & 4221 & 4197 & 4639 \\
\hline 0.46 & 0 & 2483 & 4564 & 5694 & 5093 & 4696 & 4221 & 3742 & 3491 & 3888 \\
\hline 0.48 & 0 & 2517 & 4628 & 5784 & 5186 & 4758 & 4197 & 3491 & 2732 & 2824 \\
\hline 0.5 & 0 & 2687 & 4945 & 6196 & 5630 & 5204 & 4639 & 3888 & 2824 & -3983 \\
\hline
\end{tabular}

Table 5. Torsion moment values in relation to value $-q b^{2} \times 10^{-6}$ for a square Reissner plate with relative thickness $h=0.3$ (CCCC plate, $q=$ const).

\begin{tabular}{|c|c|c|c|c|c|c|c|c|c|c|}
\hline \multirow{2}{*}{$\boldsymbol{y}$} & \multicolumn{10}{|c|}{$\boldsymbol{x}$} \\
\cline { 2 - 11 } & 0 & 0.1 & 0.2 & 0.3 & 0.4 & 0.42 & 0.44 & 0.46 & 0.48 & 0.5 \\
\hline 0 & 0 & 0 & 0 & 0 & 0 & 0 & 0 & 0 & 0 & 0 \\
\hline 0.1 & 0 & 1125 & 2047 & 2578 & 2691 & 2696 & 2714 & 2759 & 2849 & 3011 \\
\hline 0.2 & 0 & 2047 & 3729 & 4709 & 4928 & 4940 & 4977 & 5064 & 5238 & 5543 \\
\hline 0.3 & 0 & 2578 & 4709 & 5941 & 6170 & 6182 & 6231 & 6354 & 6595 & 7014 \\
\hline 0.4 & 0 & 2691 & 4928 & 6170 & 6000 & 5871 & 5794 & 5840 & 6089 & 6602 \\
\hline 0.42 & 0 & 2696 & 4940 & 6182 & 5871 & 5663 & 5488 & 5441 & 5640 & 6173 \\
\hline 0.44 & 0 & 2714 & 4977 & 6231 & 5794 & 5488 & 5171 & 4953 & 5027 & 5570 \\
\hline 0.46 & 0 & 2759 & 5064 & 6354 & 5840 & 5441 & 4953 & 4459 & 4226 & 4727 \\
\hline 0.48 & 0 & 2849 & 5238 & 6595 & 6089 & 5640 & 5027 & 4226 & 3342 & 3468 \\
\hline 0.5 & 0 & 3011 & 5543 & 7014 & 6602 & 6173 & 5570 & 4727 & 3468 & -9968 \\
\hline
\end{tabular}




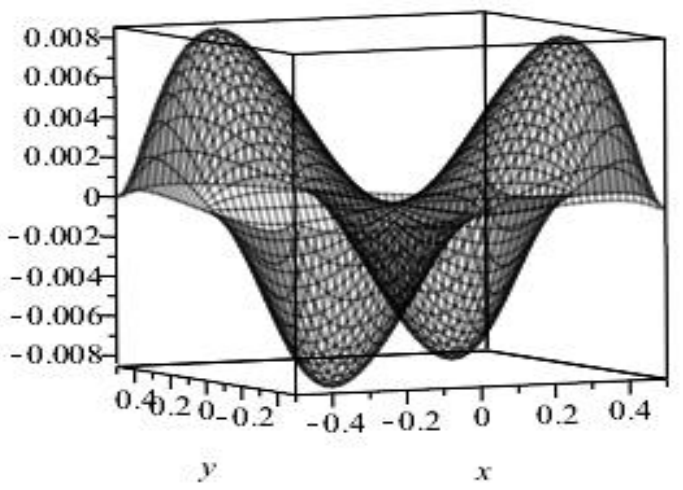

Fig. 1. Distribution diagram of torsion moments $M_{x y}$ for a clamped square Reissner plate with relative thickness $h=0.01$.

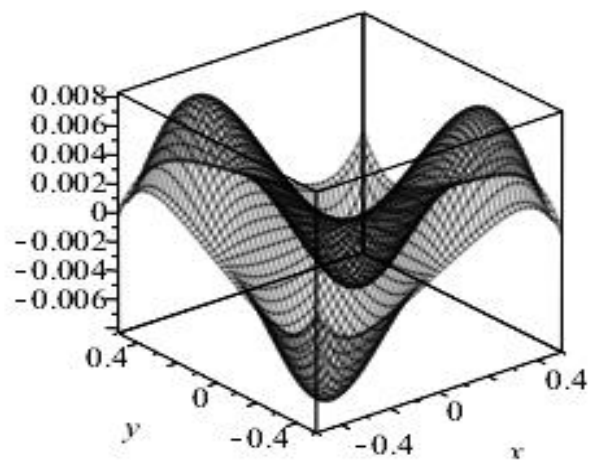

Fig. 2. Distribution diagram of torsion moments $M_{x y}$ for a clamped square Reissner plate with relative thickness $h=0.05$.

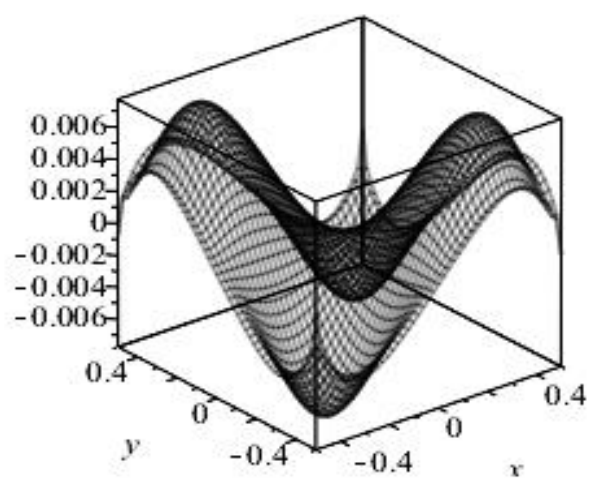

Fig. 3. Distribution diagram of torsion moments $M_{x y}$ for a clamped square Reissner plate with relative thickness $h=0.1$. 


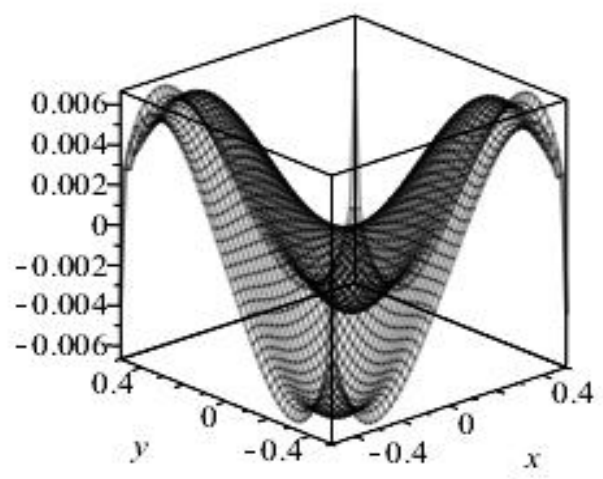

Fig. 4. Distribution diagram of torsion moments $M_{x y}$ for a clamped square Reissner plate with relative thickness $h=0.2$.

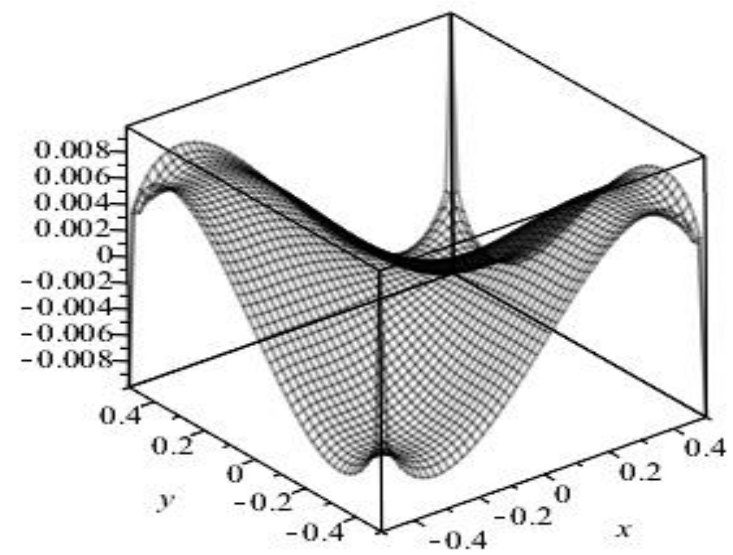

Fig. 5. Distribution diagram of torsion moments $M_{x y}$ for a clamped square Reissner plate with relative thickness $h=0.3$.

\section{Discussion}

For square plates with relative thickness $\mathrm{h}=0.01,0.05,0.1,0.2$, minimum values of torsion moment Mxy are achieved at point $(0.3,0.3)$ and are $-0.008483,-0.008257,-0.007689$, 0.006558 respectively, i.e. absolute extremum values decrease with the increase of relative thickness. Maximum values are achieved at corner point $(0.5,0.5)$ and are 0.000021 , $0.000111,0.000712,0.003983$ respectively, i.e. they grow with the increase of thickness. That said, minimum values change gradually, whereas values at the corner point increase dramatically, producing peak values. This can be seen in the corresponding diagrams and substantiates theoretical conclusions about the infinity of torsion moments at the corner points. A Reissner plate with relative thickness $h=0.01$ is similar to the Kirchhoff plate $(\mathrm{h} \approx$ 0 ) in that torsion moments at the corner points are practically zero. This is where the main feature of the refined Reissner theory becomes apparent.

With thickness $\mathrm{h}=0.3$, minimum values are achieved at two points with coordinates $(0.3$, $0.5)$ and $(0.5,0.3)$ and are -0.007014 . Maximum values 0.009968 are achieved at corner point $(0.5,0.5)$. 
The behavior pattern of the distribution diagrams of torsion moments shows that the corner points of the Reissner plate are special points, at which torsion moments are infinite. Consequently, the corresponding shear stresses $\tau x y$ are also infinite.

Now let us compare normal and shear stresses at the critical points of the plate. For this purpose, let us use the results of work [20], which calculates bending moments Mx for the similar Reissner plates.

Bending moment $\mathrm{Mx}$ at point $(0.3,0.3)$ with thickness $\mathrm{h}=0.05$ equals [20] -0.00185 , achieving maximum value equal to 0.05067 at point $(0.5,0)$. Comparison with Mxymax $(0.3$, $0.3)=-0.008257$ shows that at this point the torsion moment is approximately 4.5 times higher than the bending moment but 6.1 times lower than the maximum bending moment.

Bending moment $\mathrm{Mx}$ at point $(0.3,0.3)$ with thickness $\mathrm{h}=0.1$ equals [20] -0.00197 , achieving maximum value equal to 0.04894 at point $(0.5,0)$. Comparison with Mxymax $(0.3$, $0.3)=-0.007689$ shows that at this point the torsion moment is approximately 3.9 times higher than the bending moment but 6.4 times lower than the maximum bending moment.

Bending moment $\mathrm{Mx}$ at point $(0.3,0.3)$ with thickness $\mathrm{h}=0.2$ equals [20] -0.002823 , achieving maximum value equal to 0.04458 at point $(0.5,0)$. Comparison with Mxymax $(0.3$, $0.3)=-0.006558$ shows that at this point the torsion moment is approximately 2.3 times higher than the bending moment but 6.8 times lower than the maximum bending moment.

Bending moment $\mathrm{Mx}$ at point $(0.5,0.3)$ with thickness $\mathrm{h}=0.3$ equals [20] -0.002572 , achieving maximum value equal to 0.04049 at point $(0.5,0)$. Comparison with Mxymax $(0.5$, $0.3)=-0.007014$ shows that at this point the torsion moment is approximately 2.7 times higher than the bending moment but 5.8 times lower than the maximum bending moment.

Stresses relate to each other the same way as they are calculated by multiplying moments by $6 / \mathrm{h} 2$.

\section{Conclusion}

1. An analytical expression for torsion moments $M_{x y}$ for a rectangular Reissner plate with clamped edges is obtained and examined.

2. Torsion moments along the horizontal of a square plate are calculated, and the corresponding $3 \mathrm{D}$ distribution diagrams are built for different realtive thicknesses of the plate.

3. Changes in torsion moments depending on plate thickness are analyzed.

4. It is analytically substantiated and numerically confirmed that the corner points of the Reissner plate are special points, at which torsion moments are infinte, whereas in Kirchhoff plates they are zero.

5. Comparative analysis of normal and shear stress values is performed.

The authors express their gratitude to the government for the financial support of this research. The authors would like to gratefully acknowledge the unknown reviewers for their review and helpful comments.

\section{References}

1. E. Reissner, J. Appl. Mech. 12, 69-77 (1945)

2. R.D. Mindlin, J. Appl. Mech. 18, 31-38 (1951)

3. S. Timoshenko, S. Woinowsky-Krieger, Theory of Plates and Shells (McGraw-Hill Book Company, New York, Toronto, London, 1959)

4. S.A. Ambartsumyan, Theory of Anisotropic Plates (New York, Technomic Publishing Co., 1970) 
5. K. Vijayakumar, Arch. Appl. Mech. 81, 1717-1724 (2011) DOI: 10.1007/s00419-0110513-4

6. P.E. Tovstik, T.P. Tovstik, Doklady Physics. 8, 389-392 (2014) DOI: $10.7868 / \mathrm{S} 0869565214240128$

7. J.M. Martínez Valle, P. Martínez-Jiménez, Lat. Am. J. Solids Struct. 12, 295-316 (2015) DOI: 10.1590/1679-78251275

8. K. Nareen, R.P. Shimpi, Proc. Inst. Mech. Eng. 203-210, 1989-1996 (2015) DOI: 10.1177 / 0954406214563739

9. I. Senjanović, N. Vladimir, M. Tomić, Mech. Res. Commun. 73, 31-38 (2016) DOI.org/10.1016/j.mechrescom.2016.02.005

10. H.T. Thai, T.-K. Nguyen, T.P. Vo, T. Ngo, Compos. Struct. 171, 277-285 (2017)

11. N.F. Morozov, P.E. Tovstik, T.P. Tovstik, Mech. Solids. 51, $527-537$ (2016) DOI: $10.3103 / \mathrm{S} 0025654416050034$

12. K. Hassan, S. Guirguis, H. El-Hamouly, Afr. J. Eng. Res. 5, 7-17 (2017)

13. K. Shirakawa, Mech. Res. Commun. 10, 205-211 (1983)

14. Z. Xu, Acta Mech. Sinica-PRC. 2, 185-192 (1986)

15. Z. Xu, Int. J. Num. Meth. Eng. 33, 963-973 (1992)

16. O.C. Zienkiewicz, Z. Xu, L.F. Zeng, A. Samuelsson, N-E. Wiberg, Int. J. Num. Meth. Eng. 36, 3043-3056 (1993)

17. R. Ayad, G. Dhatt, J.L. Batoz, Int. J. Num. Meth. Eng. 42, 1149-1179 (1998)

18. S. Weiming, Y. Guangsong, Appl. Math. Mech. (Engl. Edit.) 20, 193-199 (1999)

19. H.R. Dhananjaya, Electr. J. Struct. Eng. 10, 1-10 (2010)

20. M.V. Sukhoterin, S.O. Baryshnikov, T.P. Knysh, Magaz. Civ. Eng. 76, 225-240 (2017) DOI: 10.18720/MCE.76.20 